\title{
Econometric Reviews Honors Esfandiar Maasoumi
}

\author{
Peter C. B. Phillips*and Aman Ullah ${ }^{\dagger}$
}

February 24, 2017

This Special Issue of Econometric Reviews (ER) is a tribute to Esfandiar Maasoumi for his longstanding contributions to the econometrics profession as an outstanding researcher, a revered teacher and supervisor, and a journal editor of three decades all of which manifests his extra-ordinary devotion to the discipline of econometrics and to its growing community of students and scholars. Essie, as he is universally known to his friends, colleagues, and the international community, took on the editorship of ER in 1987. At that point, as (very) senior members of the profession will remember, the journal had a paperback cover, no formal typesetting, only two small issues a year, and was no more than a review journal.

Much has changed in the intervening years. ER is now one of the top five core journals in econometrics with a distinguished editorial board, a strong track record of path-breaking publications, and the backing of a major publishing house. As Editor, Essie has guided the path of ER through these changes and built a publishing culture that has brought ER into the front rank of journals. In an age where many journal editor communications read like automated mechanical exercises, Essie's editorial letters show sensitivity to authors, empathy for the research effort that has gone into a paper, and an understanding of its subject, history, and motivation.

This Special Issue honors Essie for his sustained guidance of ER over the past three decades, for his scholarship and learning that have contributed to our knowledge of econometrics and its limitations, and for his unwavering support for the econometrics community. Thanks to its many contributors, this Issue has an impressive range of new research that covers many of the ongoing areas of econometrics, including Essie's own past and recent work.

Essie's long career began as a lecturer in econometrics at the University of Birmingham in the UK as he was finishing his Ph.D at London School of Economics under Denis Sargan in 1977. In 1978 he moved to the United States, first at the University of Southern California, then after spells teaching at Iowa, Indiana and Southern Methodist Universities, he joined Emory University where he

\footnotetext{
* Yale University, University of Auckland, University of Southampton, and Singapore Management University.

${ }^{\dagger}$ University of California Riverside
} 
currently serves as Arts and Sciences Distinguished Professor. Over his career, Essie has collaborated and co-authored with an unusually large number of researchers across the discipline, many of whom have joined us with contributions in enthusiastic support to this Special Issue of ER.

\section{Research Contributions}

Those of us who are fortunate enough to know Essie well are aware that he is widely read with strong interests in the philosophy and history of science as well as his more focused research concerns in economics and econometrics. These concerns are themselves catholic and include many branches of econometric theory, both microeconomic and macroeconomic, as well as information theory, forecasting, and policy analysis. Of special importance in economics is his work on measuring inequality and poverty, especially in multi-dimensions, and his associated work on program evaluation and treatment effect analysis. Reflecting the breadth of these interests, he has published widely in the leading journals of economics and he appears prominently in various halls of fame rankings in econometrics.

Essie's earliest work grew from his Ph.D thesis and led to several papers that focused on uncertain and mis-specified models for inference and forecasting. This work was originally cast within the reduced form framework of simultaneous equations models that formed the core of econometric analysis and modelling in the 1970s. Several strands of thinking characterize that early work and recur throughout his later work: (i) practical models are inevitably mis-specified and must be accommodated through appropriate econometric methodology; (ii) full distributions of outcomes rather than simple functions of these distributions should be accounted for in analysis and application; (iii) non-normality of economic and financial data should be respected; (iv) statistical solutions must be devised to embody these characteristics, such as test-based shrinkage and combination methods; (v) provision of frequentist, Bayesian, and information theoretic (IT) results may all be useful; and (vi) interpretations of econometric methods and why they may be expected to work or to fail often provide valuable insights.

The early Econometrica paper (Maasoumi, 1978) exemplifies many of these features, constructing as it does a Stein-rule type shrinkage estimator by combining restricted and unrestricted estimators of the parameters of a reduced form equation, with consequent advantages in tail behavior, in forecasting, and in the importation of economic theory-based prior information. Another early example is his paper in Journal of Econometrics (Maasoumi, 1980) on generalized ridge-type shrinkage estimation. The papers by Phillips (2018) and by Hansen (2018) in this Special Issue focus on these ideas and their respective contributions. Essie has recently returned to this area of research (with Caner and Riquelm, 2018) analyzing the problem of excessive numbers of instrumental variables and/or moments and how to use methods like Lasso to resolve these problems in a general GMM context. Also relevant in this connection of combining methods is his ongoing work on aggregating models, all of which are mis-specified, where IT methods are employed. 
The adoption of IT concepts and techniques reflects the availability and naturalness of distribution-based metrics in an IT framework, which facilitates analysis of distributions of outcomes with or without theory-based models. Essie has become a leader in the field of IT innovation as it applies within economics, conceptualizing objects of economic interest with IT-based econometric criteria and techniques that were first developed in communication theory. These techniques have allowed him to formulate many popular hypotheses in their naturally intended forms (such as dependence, goodness of fit, predictability, symmetry, and reversibility) and to provide rigorous tests of them based on entropic functions.

Seeking ways to rank distributed outcomes by means of general stochastic dominance methods is another manifestation of Essie's concern for the development of evaluative criteria that go beyond simple moment-function (such as mean, variance or mean squared error) assessments. The paper with Linton and Whang in the Review of Economic Studies (2005) is an influential and highly cited paper, especially in finance, within this paradigm. The paper constructs a useful framework for subsequent empirical work by providing extended Kolmogorov-Smirnov tests of stochastic dominance of arbitrary order, in which observations may be serially dependent and where ranked prospects are allowed to be dependent, using subsampling methods.

Essie's concerns to employ statistically rigorous and uniform/dominance rankings arose from his work on indices for measuring inequality, poverty, and mobility; and he is one of the leaders in developing the empirical/statistical income inequality literature, with influential works on multidimensional measures of well-being, mobility, and poverty. His works have helped to bring rigorous inferential tools to this field and his measures of aggregation and inequality in multiple dimensions are a gold standard in the field, together with his joint work on stochastic dominance The multidimensional parts of his work on welfare analysis were inspired by the early influence of Amartya Sen, one of his professors at the LSE. They represent a statistical embodiment of some of Sen's ideas and concepts, have been applied widely, and are among the most cited work in the area. His 1986 Econometrica paper is highly cited for analyzing multi-attribute characteristics. Many other proposed measures of inequality or poverty in multiple dimensions develop from the measures developed in that paper. Related measures of mobility were advanced in joint work (1986, 1989, 1990) with a former student (Zandvakili) using aggregation techniques of the 1986 paper to define and measure permanent or long run incomes that have been highly sought in the field of mobility. The aggregate measures of well-being arising from these measures are known to subsume and/or dominate many other popular measures, including the United Nations indices of well-being at the country level.

Finally, in concordance with the above thinking on measurement, Essie's work on program evaluation and treatment effects characteristically emphasizes the whole distribution of outcomes, rather than an 'average treatment effect' or other summary measure. In this line of work, he has used information measures to 'define twins' - that is 'matches' for the identification of unobserved coun- 
terfactual outcomes in order to create entirely missing samples. He has used these ideas and techniques, as well as stochastic dominance tests, to assess the impact of class size, marriage, and union membership on wage outcomes. More recent work has analyzed the gap between the distributions of wages for men and women, and studied counterfactual distributions of women, citizen and non citizen workers, and workers with different tenure/work attachment characteristics. Many examples of this line of work appear in joint papers with Millimet and students from SMU and Emory.

\section{Research Papers in this Special Issue}

This special issue includes 25 papers contributed by Essie's many friends, mentors, colleagues, teachers, and co-authors. All papers have been reviewed in the usual manner by at least two independent referees and held to the usual Econometric Reviews standard. In our discussion that follows, the papers that were accepted and are now included in this Special Issue are broadly classified into three areas: time series and forecasting; information theory; treatment effects and general econometric theory.

Within the time series and forecasting group, Duffy and Hendry analyze asymptotics and simulate the impact of measurement errors on parameter estimates and tests in a bivariate co-integrated system with trends and location shifts reflecting many turbulent events that have occurred historically. The measurement errors considered in the paper are from data that span long time periods which, the authors demonstrate, can lead to series that are integrated rather than stationary, with consequent effects on the limit theory and bias. Diebold and Shin introduce a new stochastic error distance (SED) as a measure of forecast accuracy which is based on distance of the forecast-error of the cumulative distribution function from the unit step function at the origin. Standard predictive loss functions are shown to be weighted SED's and they suggest shifting the evaluative focus from conditional mean forecasts to conditional median forecasts. The paper by Catani, Terasvirta, and Yin provides an LM test for misspecification in a conditional constant correlation GARCH model which gives good performance in small samples as confirmed in simulations. Medeiros and Mendes study the validity of an adaptive LASSO procedure in estimating a stationary autoregressive distributed lag model with non-Gaussian GARCH innovations when the number of regressors potentially exceeds the sample size, but only few regressors are relevant. Asai and McAleer introduce a modified covariance estimator to study the impact of jumps in forecasting co-volatility, accommodating leverage effects. Empirical results are provided for three stocks traded on the New York Stock Exchange. The paper by Astill, Harvey, Leybourne, and Taylor propose a sub-sampling test strategy to explore the issue of detecting explosive behavior in time series data when an explosive episode is both ongoing at the end of sample and of finite length. There are two objectives in the paper by Hall, Osborn, and Sakkas: first to show that the number of estimated break points and the number of regression parameters have different effects on the expectation of the residual sum of squares, and second to propose a statistic for testing the joint hypothesis that the break points occur at 
specified points in the sample. Kiefer's paper proposes a Bayesian approach to default rate estimation and illustrates this using prior distributions generated from an experienced industry expert. This framework is then used to assess predictability of default rates.

In the information theoretic group, the paper by Hong, Wang, Zhang, and Wang provides a numerically integrated non-parametric entropy-based test for serial dependence and shows its performance betters that of the Hong and White test based on sample-average entropy estimation, so that it is asymptotically more efficient. The Golan and Ullah paper considers a regression model with interval data and provides an inferential procedure based on entropy maximization subject to multi-dimensional moment conditions, where the entropy is defined over discretized intervals. The Shoja and Soofi paper proposes an information theoretic framework that builds a mixture model for combining density forecasts, which is used to study uncertainty and disagreement among economic forecasters.

The final group of papers involve several topics in econometric theory and treatment effects. The paper by Phillips studies reduced form prediction, developing both exact small sample and asymptotic distributions for reduced form estimators and predictors. Using these results, the paper develops new weak instrument asymptotics, showing the advantages for prediction of the shrinkage induced by the use of structural information, via the partially restricted reduced form mechanism. Following Maasoumi's approach on combined estimation, Hansen's paper develops a combined estimator of a structural equation parameter by combining 2SLS and OLS estimators. The proposed estimator is a Stein-type shrinkage estimator and is asymptotically more efficient than 2SLS under certain conditions. In the context of a fixed effect panel model, Baltagi, Kao and Wang study the asymptotic power of a proposed sphericity test under the alternative of weak and strong factors, showing that the test is inconsistent when the factors are weak. Hsiao and Zhou study asymptotic properties of Arellano-Bond-type GMM estimation, showing biased estimation when using first differences of the dynamic panel and either all lags or a single lag as instruments, but with forward demeaning biased estimation when using instruments with all lags but unbiased estimation when using only a fixed number of lags as instruments. In another panel model paper Al-Sadoon, Li, and Pesaran consider short $(T)$, wide $(N)$ panels, allowing fixed effects to be arbitrarily correlated with the covariates. In this scenario they propose GMM estimators that are consistent and asymptotically normal at the $\sqrt{N}$ rate. Continuing with GMM methods, Antoine and Renault demonstrate improvements in the efficiency of GMM under certain conditions when additional weaker moment conditions are available and utilized. Han and Caner work with a factor model and develop consistent methods to estimate the number of factors when the error terms have strong correlations in the cross-sectional dimension. The $\mathrm{Li}, \mathrm{Li}$, and Racine paper provides a computationally efficient data-driven crossvalidation procedure to select smoothing parameters for nonparametric estimation of a cumulative distribution with mixed covariates (discrete/continuous). In another nonparametric paper $\mathrm{Li}$, Liu, and $\mathrm{Li}$ develop a nonparametric Knn 
regression estimator subject to a monotonicity restriction which differs from the Hall-Huang monotone kernel approach. The paper by Amsler, O'Donnell, and Schmidt develops a stochastic metafrontier distance, which is formed by the envelope of the group-specific frontiers and which differs from earlier work giving a metafrontier distance based on the deterministic portion of the frontier. The paper by Davidson provides diagnostic techniques capable of evaluating bootstrap performance in specific cases, that include time series models with serial correlation of unknown form, a case where the bootstrap is typically less reliable. Bao, Ullah, and Wang develop the exact probability distribution of the mean reversion parameter estimator in an Ornstein-Uhlenbeck diffusion process. Finally, the paper by Fan and Manzanares systematically studies identification of the average treatment effect on the treated when differences in differences is used and the post-treatment status is unknown for the pre-treatment sample.

\section{References}

Hansen, B. E. (2018). "A Stein-Like 2SLS Estimator", Econometric Reviews, 2018 (forthcoming - This Issue)

Linton, O., E. Maasoumi and Y-J. Whang (2005). "Consistent Testing for Stochastic Dominance Under General Sampling Schemes," Review of Economic Studies, 72, 735-765.

Maasoumi, E. (1978). "A Modified Stein-like Estimator for the Reduced Form Coefficients of Simultaneous Equations," Econometrica 46, 695-703.

Maasoumi, E. (1980). "A Ridge-like Method for Simultaneous Estimation of Simultaneous Equations," Journal of Econometrics, 12, 161-176.

Maasoumi, E. (1986). "The Measurement and Decomposition of Multi-dimensional Inequality," Econometrica, 54, 991-998.

Maasoumi, E., M. Caner and J. A. Riquelme (2018). "Moment and IV Selection Approaches: A Comparative Simulation Study," Econometric Reviews (forthcoming).

Maasoumi, E. and S. Zandvakili (1986). "A Class of Generalized Measures of Mobility with Applications," Economics Letters, 22, 97-102.

Maasoumi, E. and S. Zandvakili (1989). "Mobility Profiles and Time Aggregates of Individual Incomes," Research on Economic Inequality, 1, 195218.

Maasoumi, E. and S. Zandvakili (1990). "Generalized Entropy Measures of Mobility for Different Sexes and Income Levels," Journal of Econometrics, 43, 121-133.

Phillips, P. C. B. (2018). "Reduced Forms and Weak Instrumentation", Econometric Reviews, 2018 (forthcoming - This Issue) 